\title{
Sucht und elterliche Stressbelastung: Das spezifische Belastungserleben in der Kindererziehung von alkoholabhängigen Müttern und substituierten opiatabhängigen Müttern
}

\author{
C. Kröger \\ M. Klein \\ I. Schaunig
}

\author{
Addiction and Parental Stress: Specific Burden Experienced during Child \\ Parenting by Alcohol Dependent Mothers and Opiate Maintained Mothers
}

\section{Zusammenfassung}

Um angemessene Hilfsangebote für Eltern mit Suchterkrankungen entwickeln zu können, ist es notwendig, die besonderen Schwierigkeiten dieser Zielgruppe zu kennen. Daher wurden im Rahmen der vorliegenden Studie die spezifischen Belastungen in der Kindererziehung von substanzabhängigen Müttern ermittelt und mit denen von nicht suchtbelasteten Müttern verglichen. Im Einzelnen wurde das Stresserleben von 51 alkoholabhängigen Müttern, 92 substituierten opiatabhängigen Müttern und 75 Müttern ohne Suchtprobleme anhand des „Parenting Stress Index“ (PSI) von Abidin erfasst. Insgesamt zeigt sich eine beeindruckende Ähnlichkeit im Belastungsmuster der alkohol- und der opiatabhängigen Mütter in Substitutionsbehandlung, die sich vor allem darin ausdrückt, dass suchtkranke Frauen Schwierigkeiten in der Erziehung eher auf eigene Probleme bzw. Defizite und weniger auf Eigenschaften oder Verhaltensweisen ihres Kindes zurückführen. Die Mütter mit Suchtstörung erleben ausgeprägte Zweifel an ihren erzieherischen Fähigkeiten und sind sozial isolierter und stärker als nicht suchtbelastete Mütter durch depressive Symptome und äußere Lebensumstände belastet. Die Ergebnisse werden im Hinblick auf Implikationen für die Gestaltung entsprechender Interventionsangebote diskutiert.

\section{Schliüsselwörter}

Sucht · Erziehungskompetenz · Kindererziehung · alkoholabhängige Mütter · opiatabhängige Mütter in Substitution · Parenting Stress Index

\section{Abstract}

In order to develop well-suited intervention and support measures for addicted parents it is necessary to know the specific problems of this group very well. Therefore, in this study specific parental stressors of addicted mothers were investigated in depth. The stress factors of child rearing mothers with diagnoses of different AOD disorders were compared to those of mothers without AOD problems. In detail, parental stress was measured by applying Abidin's "Parenting Stress Index" (PSI) to 51 alcohol dependent mothers in inpatient treatment, 92 mothers in outpatient methadone maintenance treatment, and 75 mothers without AOD problems. All in all, the results show an astonishing similarity concerning amount and intensity of stress reported by alcohol and drug dependent mothers. Especially, self reported stress of feelings of inadequacy as mother was reported by both groups. Mothers with AOD problems seem to attribute higher rates of parenting stress to own problems and deficits and less to specific traits or behavioral problems of their child. Mothers with AOD problems report intensive doubts concerning their own parental competencies, are much more isolated, more depressed and strained by external circumstances of their living reality than mothers without AOD problems. The results are finally discussed concerning their impact on treatment and intervention systems.

Key words

Addiction - parental competence - parenting - alcohol dependent mothers - mothers in methadone maintenance treatment . Parenting Stress Index 
Die Bedeutung der Erziehung in der Familie und die Frage, wie Eltern wirksam unterstützt werden können, ist in den letzten Jahren sowohl in fachlicher als auch in politischer und gesamtgesellschaftlicher Hinsicht verstärkt in den Mittelpunkt des Interesses gerückt. Verschiedene renommierte Familienforscher heben die zentrale Rolle der Familienbeziehungen und der familialen Erziehung für die Persönlichkeitsentwicklung von Kindern und Jugendlichen hervor [1 - 3] und verweisen auf den engen Zusammenhang zwischen destruktiven familialen Interaktionsmustern und Entwicklungsauffälligkeiten der Kinder [4,5]. Dementsprechend setzt sich auch der Wissenschaftliche Beirat für Familienfragen beim Bundesministerium für Familie, Senioren, Frauen und Jugend (BMFSFJ) [6] in einem aktuellen Gutachten mit der Bedeutung familialer Beziehungs- und Erziehungskompetenzen auseinander und zeigt Maßnahmen auf, wie entwicklungsförderliches Elternund Erzieherverhalten vermittelt werden kann. Darüber hinaus wird im 12. Kinder- und Jugendbericht des BMFSFJ [7] betont, dass sich die Bundesregierung im ersten Jahrzehnt des neuen Jahrtausends zum Ziel gesetzt hat, die Rahmenbedingungen für das Aufwachsen der nachfolgenden Generation zu verbessern und dass hierfür nicht zuletzt die Eltern unterstützt werden müssen. Auf gesamtgesellschaftlicher Ebene spiegelt unter anderem das breite Interesse an Fernsehformaten wie der „Super Nanny“ wider, dass etliche Eltern verunsichert sind und ein Bedürfnis nach Orientierung bei der Erziehung ihrer Kinder haben. Trotz der allgemeinen Präsenz der Thematik spielen diejenigen Zielgruppen, die eine entsprechende Unterstützung besonders dringend benötigen, in der gegenwärtigen Diskussion meist nur eine marginale Rolle. $\mathrm{Zu}$ diesen „vergessenen Eltern“ gehören insbesondere substanzabhängige Mütter. Obgleich mittlerweile zahlreiche, zum Teil recht unterschiedliche Elternkurskonzepte und familienbasierte Interventionsprogramme entwickelt wurden (Überblick bei $[8,9])$, gibt es kaum Angebote, die auf den besonderen Hilfebedarf von Müttern mit gravierenden Suchtproblemen abgestimmt sind. Gleichzeitig ist davon auszugehen, dass suchtbelastete Frauen mit den herkömmlichen Elternkursen (wie z.B. Triple P, STEP oder Starke Eltern - starke Kinder ${ }^{\circledR}$, vgl. [9]) nicht erreicht werden; die Gründe hierfür sind sicherlich vielfältig, wobei Schamgefühle und Angst vor Stigmatisierung eine nicht unerhebliche Rolle spielen dürften. Letztlich wären diese Angebote jedoch auch nur begrenzt sinnvoll, da davon auszugehen ist, dass suchtkranke Mütter teilweise andere und sehr spezifische Bedürfnisse aufweisen und ebenso differente Anforderungen bei der Erziehung ihrer Kinder erleben.

Eine wesentliche Voraussetzung für die Entwicklung und Etablierung angemessener Unterstützungsangebote für suchtbelastete Eltern besteht somit darin, die speziellen Problemlagen und Schwierigkeiten dieser Zielgruppe zu ermitteln. Um hier einen empirisch fundierten Beitrag zu leisten, werden im Rahmen der vorliegenden Arbeit erstmals die besonderen Belastungen in der Kindererziehung von (a) alkoholabhängigen Müttern und (b) substituierten opiatabhängigen Müttern erfasst und mit denen von nicht suchtbelasteten Müttern verglichen. Zusätzlich wird geprüft, ob und inwieweit sich das Belastungserleben von Alkoholikerinnen und opiatabhängigen Müttern in Substitutionsbehandlung unterscheidet.

Insgesamt ist davon auszugehen, dass eine professionelle Unterstützung sowohl bei alkohol- als auch bei opiatabhängigen substi- tuierten Müttern sinnvoll ist, da beide Suchterkrankungen die Erziehungskompetenz deutlich beeinträchtigen können. Immerhin zeichnet sich die Lebenssituation dieser Mütter meist durch eine Kumulation gravierender Belastungsfaktoren aus, wie z.B. nicht bewältigte eigene Kindheitstraumata, instabile und konfliktreiche Partnerschaften, gesundheitliche Komplikationen und Armut, sodass für die Kinder eine ernstzunehmende Entwicklungsgefährdung besteht. Dementsprechend dokumentieren verschiedene Forschungsarbeiten, dass Kinder aus suchtbelasteten Familien ein deutlich erhöhtes Risiko für Verhaltensauffälligkeiten und psychische Störungen im Kindes- und Jugendalter aufweisen, wobei die stärkste Gefährdung im Hinblick auf eine spätere eigene Suchtproblematik besteht [10-13].

Gleichwohl gilt zu bedenken, dass sich die psychosoziale Situation opiatabhängiger Mütter - nicht zuletzt aufgrund der Illegalisierung der Substanz und den damit verbundenen, häufig langfristig wirksamen Belastungen (wie z.B. Kriminalisierung, hohe Verschuldung, fehlende soziale Integration) - in der Regel weitaus besorgniserregender und kritischer darstellt (vgl. auch [10]) als die alkoholabhängiger Mütter.

Auch epidemiologische Aspekte unterstreichen die Notwendigkeit, entsprechende Unterstützungsangebote für alkoholabhängige und substituierte opiatabhängige Mütter zu konzipieren: Nach Schätzungen der Bundeszentrale für gesundheitliche Aufklärung (BzgA) [14] kann davon ausgegangen werden, dass jedes Jahr mindestens 8000 Kinder von alkoholabhängigen Müttern geboren werden. Bei den opiatabhängigen Frauen ist insbesondere durch die Etablierung der Substitutionsbehandlung eine deutliche $\mathrm{Zu}$ nahme der Geburtenrate zu verzeichnen: Ca. 40000 bis 50000 Kinder wachsen bundesweit mit mindestens einem opiatabhängigen Elternteil auf [15].

\section{Methode}

\section{Stichprobe}

Insgesamt wurden 218 Mütter (51 alkoholabhängig, 92 opiatabhängig und in Substitutionsbehandlung sowie 75 ohne Suchtprobleme) zu ihrem Belastungserleben in der Kindererziehung befragt. Die alkoholkranken Mütter wurden im Rahmen einer Diplomarbeit [16] über drei unterschiedliche Fachkliniken rekrutiert und befragt. Die opiatabhängigen Mütter konnten über verschiedene Substitutionsambulanzen in NRW zur Teilnahme an der Studie gewonnen werden. Die Gruppe der Mütter ohne Suchtprobleme, die als Kontrollgruppe fungiert, wurde zur Hälfte $(n=37)$ im Rahmen der genannten Diplomarbeit rekrutiert, die andere Hälfte $(\mathrm{n}=38)$ wurde über Kindertagesstätten und Familienbildungsstätten angesprochen.

In Tab. 1 sind die wichtigsten soziodemographischen Merkmale zusammengefasst. Die drei Gruppen wurden bezüglich der erfassten Merkmale nicht parallelisiert, da an der Studie gezielt naturalistische Gruppen aus ihrem jeweiligen Sozialraum teilnehmen sollten. Demzufolge ergeben sich für die verschiedenen soziodemographischen Charakteristika recht ausgeprägte Differenzen zwischen den Gruppen. Die opiatabhängigen substituierten Mütter sind mit durchschnittlich 32,4 Jahren signifikant jünger als die 
Tab. 1 Soziodemographische Merkmale der alkoholabhängigen Mütter (Alk-M; $n=51$ ), der substituierten opiatabhängigen Mütter (Sub-M $\mathrm{n}=92$ ) und der nicht suchtbelasteten Mütter (Unb-M; n=75)

\begin{tabular}{lrrrrrrr}
\hline & \multicolumn{1}{c}{ Alk-M } & \multicolumn{3}{c}{ Sub- $\boldsymbol{M}$} & \multicolumn{3}{c}{ Unb-M } \\
& $\boldsymbol{M}$ & (SD) & $\boldsymbol{M}$ & (SD) & $\boldsymbol{M}$ & (SD) \\
\hline Alter der Mutter & 39,3 & $(6,9)$ & 32,4 & $(6,3)$ & 36,7 & $(6,6)$ \\
\hline Kinderzahl & 1,9 & $(1,0)$ & 1,8 & $(0,9)$ & 1,6 & $(0,7)$ \\
\hline Alter des ältesten Kindes & 12,0 & $(7,2)$ & 7,1 & $(6,3)$ & 6,9 & $(8,5)$ \\
& & & & & & \\
& $\%$ & $(\mathrm{n})$ & $\%$ & $(\mathrm{n})$ & $\%$ & $(\mathrm{n})$ \\
Familienstand: & & & & & & \\
ledig & 23 & $(12)$ & 42 & $(37)$ & 17 & $(13)$ \\
verheiratet & 39 & $(20)$ & 17 & $(15)$ & 72 & $(54)$ \\
verheiratet, aber getrennt lebend & 14 & $(7)$ & 11 & $(10)$ & 0 & $(0)$ \\
geschieden & 21 & $(11)$ & 30 & $(26)$ & 9 & $(7)$ \\
verwitwet & 4 & $(2)$ & 0 & $(0)$ & 1 & $(1)$ \\
erwerbstätig & 25 & $(49)$ & 9 & $(10)$ & 40 & $(53)$ \\
\hline
\end{tabular}

$\mathrm{M}=$ Mittelwert, SD = Standardabweichung, \% = prozentualer Anteil, $\mathrm{n}=$ absolute Häufigkeit.

Mütter der beiden anderen Gruppen $(F(2,207)=19,49, p \leq, 000)$. Im Hinblick auf das Alter des ältesten Kindes heben sich die alkoholabhängigen Mütter deutlich ab $(F(2,211)=9,42, p \leq, 000)$. Bezüglich des Familienstands $\left(\chi^{2}=59,63, \mathrm{df}=2, \mathrm{p} \leq, 000\right)$ und der Erwerbstätigkeit $\left(\chi^{2}=40,13, \mathrm{df}=2, \mathrm{p} \leq, 000\right)$ zeigen sich ebenfalls signifikante Unterschiede. Lediglich für die durchschnittliche Kinderzahl ergibt sich keine signifikante Differenz zwischen den drei Gruppen $(F(2,213)=2,30$, $p=$ n. s. $)$.

\section{Erhebungsinstrumente}

Zur Erfassung der elterlichen Stressbelastung wurde eine deutsche Übersetzung des Parenting Stress Index (PSI) [17] eingesetzt. Beim PSI handelt es sich um einen etablierten standardisierten Fragebogen, der mit insgesamt 120 Items das Ausmaß des Stresserlebens in den Eltern-Kind-Beziehungen und der Kindererziehung aus elterlicher Perspektive abbildet. Der PSI umfasst eine kind- und eine elternbezogene Belastungsdimension sowie einen Life-Stress-Score, der die Stressbelastung durch äußere Lebensumstände (wie z.B. Tod eines nahen Freundes oder eines Angehörigen, Trennung oder Scheidung, hohe Verschuldung etc.) widerspiegelt. Die Kinddimension besteht aus den Subskalen Ablenkbarkeit, Anpassungsfähigkeit, Verstärkung, Anforderungen an die Eltern, Stimmung und Akzeptanz. Hohe Ausprägungen der Kinddimension verweisen auf Verhaltensweisen und Temperamentsmerkmale des Kindes, die es den Eltern erschweren, ihre Rolle auszufüllen und der Erziehungsaufgabe gerecht zu werden. Die Elterndimension umfasst die folgenden Subskalen: Kompetenz, Isolation, Bindung, Gesundheit, Einschränkungen durch die Elternrolle, Depression und Partnerschaft. Hohe Werte in diesem Bereich sind als Hinweis darauf zu verstehen, dass Belastungen in der Kindererziehung eher auf eigene Schwierigkeiten und Probleme der Eltern (z.B. negatives elterliches Selbstbild, partnerschaftliche Disharmonie) zurückzuführen sind. Abidin [17] weist darauf hin, dass Eltern, die hier hohe Werte erreichen, in ihrem Selbstbild bestärkt und unterstützt werden sollten. Werden die Werte der Kinddimension und der Elterndimension aufsummiert, ergibt sich der Total-StressScore.

\section{Ergebnisse}

In Abb. 1 sind die entsprechenden PSI-Scores für die drei untersuchten Gruppen dargestellt. Diese Profildarstellung zeigt auf der linken Seite die Mittelwerte für die Subskalen und den Gesamtwert der Kind-Dimension, während die rechte Seite über die Ausprägungen auf den elternbezogenen Subskalen sowie den entsprechenden Gesamtwert informiert. Ganz rechts finden sich schließlich noch der Total-Stress- und der Life-Stress-Score. Für alle Scores gilt, dass höhere Werte auf eine stärkere Belastung verweisen. Gleichzeitig informiert die Darstellung über die jeweiligen Prozentränge (siehe die entsprechenden Perzentilskalen auf der linken und rechten Seite des Profils). Den Normbereich fasst Abidin [17] vergleichsweise weit, nämlich zwischen dem 15 . und dem 85 . Prozentrang; im Profilblatt ist der Durchschnittsbereich durch entsprechende Linien markiert.

Ein vergleichender Blick auf die Profilverläufe der drei Gruppen zeigt, dass die Werte für die nicht suchtbelasteten Mütter erwartungsgemäß fast durchgängig am niedrigsten ausfallen. Für den kindbezogenen Bereich lässt sich insgesamt festhalten, dass die Einschätzungen der Mütter aus allen drei Gruppen innerhalb der Norm liegen. Dennoch wird deutlich, dass die opiatabhängigen substituierten Mütter hier zum Teil deutlich stärkere Belastungen erleben (z. B. im Hinblick auf die Anpassungsfähigkeit ihres Kindes an neue und unbekannte Situationen) als die alkoholabhängigen und die nicht suchtbelasteten Mütter. Bezüglich des elternbezogenen Bereichs zeigt sich vor allem, dass die Profilverläufe der alkoholabhängigen und der substituierten Mütter sehr ähnlich ausfallen. Zudem sticht hier hervor, dass es mehrere Bereiche gibt, in denen die suchtbelasteten Mütter, d.h. sowohl die Alkoholikerinnen als auch die Frauen in Substitutionsbehandlung, ausgesprochen starke bis überdurchschnittlich ausgeprägte Belastungen aufweisen: Dies betrifft zum einen die Einschätzung ihrer eigenen erzieherischen Kompetenzen und zum anderen die Belastung durch äußere Lebensumstände (Life Stress). Darüber hinaus rangiert der Skalenwert für die soziale Isolation sehr nah am Cut-offWert für überdurchschnittlich starkes Stresserleben. Es wird auch deutlich, dass die suchtbelasteten Mütter im Hinblick auf elternbezogene Aspekte und Eigenschaften eine weitaus höhere Belastung aufweisen als bezüglich der kindbezogenen Merkmale. Dieses Belastungsmuster deutet darauf hin, dass suchtkranke Mütter Schwierigkeiten in der Kindererziehung eher auf sich selbst und weniger auf problematische Eigenschaften und Verhaltensweisen ihres Kindes zurückführen.

Um zu prüfen, ob zwischen den drei Gruppen signifikante Unterschiede in den PSI-Skalenwerten bestehen, wurden aufgrund ungleich großer Stichproben und heterogener Varianzen KruskalWallis-H-Tests (ein nonparametrisches Verfahren für Ranganalysen) berechnet (vgl. [18]). Bei Hinweisen auf bedeutsame Differenzen wurde anhand von Mann-Whitney-U-Tests weiterführend untersucht, welche Gruppen sich im Einzelnen signifikant voneinander unterscheiden.

In Tab. 2 sind die Resultate der Kruskal-Wallis-H-Tests zusammengefasst. Aufgrund der vergleichsweise hohen Anzahl an Einzeltests wurde eine $\alpha$-Fehler-Adjustierung nach Bonferroni $(\mathrm{p} \leq, 003)$ durchgeführt (vgl. [19]). 


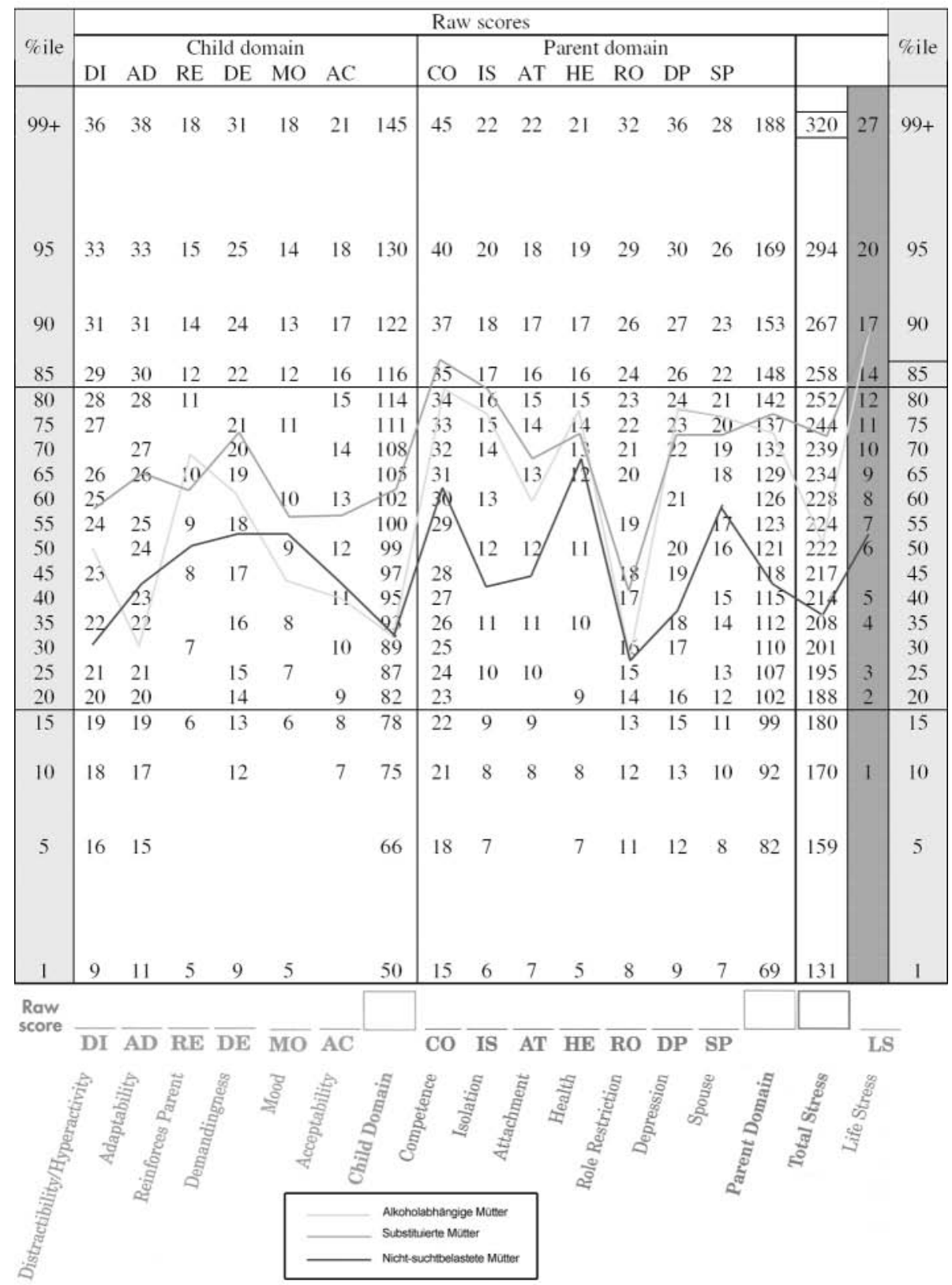

Abb. 1 PSI-Scores für die drei untersuchten Gruppen.

Im Hinblick auf die Kinddimension zeigen sich sowohl für den Gesamtwert als auch für die Subskalen Ablenkbarkeit, Anpassungsfähigkeit und Anforderungen an die Eltern signifikante Ergebnisse. Die entsprechenden Einzelvergleiche belegen (Tab. 3), dass es (mit Ausnahme der Subskala „Ablenkbarkeit“) die substituierten Mütter sind, die signifikant höhere Stresswerte erreichen als die alkoholabhängigen und die nicht suchtbelasteten Mütter. Dies bedeutet, dass die opiatabhängigen Mütter in Substitutionsbehandlung die Anpassungsfähigkeit ihrer Kinder geringer einschätzen und ihre Kinder als anstrengender und fordernder erleben als die Mütter der beiden anderen Gruppen. In Bezug auf die Ablenkbarkeit des Kindes weisen sowohl die Mütter in Substitutionsbehandlung als auch die Alkoholikerinnen eine signifikant höhere Belastung auf als die Kontrollgruppe. Im elternbezogenen Bereich zeigen sich signifikante Gruppenunterschiede hinsichtlich des Kompetenzerlebens, der sozialen Isolation, der Beeinträchtigung durch depressive Verstimmungen sowie des Gesamtwerts. Die MannWhitney-U-Tests bestätigen (Tab.3), dass das Belastungserleben der alkoholabhängigen und substituierten Mütter in diesen
Aspekten jeweils vergleichbar ausfällt, sich aber hochsignifikant von den nicht suchtbelasteten Müttern abhebt. Somit kann festgehalten werden, dass die alkoholabhängigen Mütter ebenso wie die Mütter in Substitutionsbehandlung ihre eigene erzieherische Kompetenz und ihr Elternverhalten sehr negativ einschätzen und oftmals befürchten, der Erziehungsaufgabe nicht gerecht werden zu können (vgl. auch Abb.1). Die Ergebnisse bestätigen auch, dass suchtbelastete Mütter sozial isolierter sind und in weitaus höherem Maß unter depressiven Verstimmungen leiden als nicht suchtbelastete Mütter.

Darüber hinaus spiegelt sich die besondere Belastungssituation von süchtigen Müttern im Life-Stress-Score wider, der sowohl für alkohol- als auch für die opiatabhängigen Mütter außerhalb des Normbereichs liegt und signifikant höher ausfällt als bei den befragten unbelasteten Müttern (Tab.3). Abidin [17] betont, dass eine erhöhte Belastung durch äußere Lebensumstände (hierzu gehören beispielsweise Trennung oder Scheidung, hohe Verschuldung, Tod nahestehender Menschen) im Allgemeinen mit einem 
Tab. 2 Ergebnisse der Kruskal-Wallis-H-Tests zur Prüfung auf Unterschiede in den Skalenwerten zwischen alkoholabhängigen (Alk-M; $n=51)$, substituierten (Sub-M; $n=92$ ) und unbelasteten Müttern (Unb-M; n = 75)

\begin{tabular}{lrlrrl}
\hline \multicolumn{1}{c}{$\begin{array}{l}\text { Ränge für die drei } \\
\text { Gruppen }\end{array}$} & & \\
& Alk-M & Sub-M & Unb-M & $\chi^{2}$ & P \\
\hline PSI-Skalen & 94,9 & 131,1 & 91,2 & 19,93 & $* * *$ \\
\hline Kind-Dimension & 114,0 & 125,7 & 86,6 & 16,32 & $* * *$ \\
\hline Ablenkbarkeit & 84,9 & 128,8 & 99,0 & 18,49 & $* * *$ \\
\hline Anpassungsfähigkeit & 122,1 & 115,1 & 92,8 & 8,12 & ns. \\
\hline Verstärkung & 101,6 & 127,1 & 90,2 & 15,25 & $* * *$ \\
\hline Anforderungen an die Eltern & 93,6 & 115,9 & 107,6 & 4,19 & ns. \\
\hline Stimmung & 94,1 & 124,7 & 98,1 & 10,95 & ns. \\
\hline Akzeptanz & 119,6 & 127,8 & 77,5 & 28,83 & $* * *$ \\
\hline Eltern-Dimension & 114,4 & 130,9 & 77,2 & 31,13 & $* * *$ \\
\hline Kompetenz & 117,9 & 133,7 & 71,4 & 42,70 & $* * *$ \\
\hline Isolation & 110,1 & 120,4 & 92,8 & 8,21 & ns. \\
\hline Bindung & 124,1 & 113,5 & 90,8 & 9,81 & ns. \\
\hline Gesundheit & 100,5 & 119,4 & 100,4 & 4,91 & ns. \\
\hline Einschränkungen durch die Elternrolle & 124,6 & 125,6 & 77,0 & 29,30 & $* * *$ \\
\hline Depression & 119,4 & 114,8 & 93,6 & 6,68 & ns. \\
\hline Partnerschaft & 108,0 & 132,3 & 82,6 & 25,73 & $* * *$ \\
\hline Total Stress & 127,7 & 128,9 & 62,0 & 19,93 & $* * *$ \\
\hline Life Stress & & & & & \\
\hline
\end{tabular}

Anmerkungen: Die Freiheitsgrade (df) betrugen jeweils 2.

ns. $=$ nicht signifikant, ${ }^{*}=p \leq .003$ (Bonferroni-Korrektur), ${ }^{*}=p \leq .001$, $* * *=p \leq .000$

Tab. 3 Zusammenfassung der Ergebnisse der Einzelvergleiche

\begin{tabular}{|c|c|}
\hline PSI-Skalen & Ergebnisse der Mann-Whitney-U-Tests \\
\hline Kind-Dimension & Sub-M > Alk-M**; Alk-M, Unb-M; Sub-M > Unb-M*** \\
\hline Ablenkbarkeit & Alk-M, Sub-M; Alk-M > Unb-M*; Sub-M > Unb-M*** \\
\hline Anpassungsfähigkeit & Sub-M > Alk-M ${ }^{* * *}$; Alk-M, Unb-M; Sub-M > Unb-M** \\
\hline $\begin{array}{l}\text { Anforderungen an } \\
\text { die Eltern }\end{array}$ & Sub-M > Alk-M*; Alk-M, Unb-M; Sub-M > Unb-M*** \\
\hline Eltern-Dimension & Sub-M, Alk-M; Alk-M > Unb-M***; Sub-M > Unb-M*** \\
\hline Kompetenz & Sub-M, Alk-M; Alk-M > Unb-M***; Sub-M > Unb-M*** \\
\hline Isolation & Sub-M, Alk-M; Alk-M > Unb-M***; Sub-M > Unb-M*** \\
\hline Depression & Sub-M, Alk-M; Alk-M > Unb-M***; Sub-M > Unb-M*** \\
\hline Total Stress & Sub-M > Alk-M*; Alk-M > Unb-M*; Sub-M > Unb-M*** \\
\hline Life Stress & Sub-M, Alk-M; Alk-M > Unb-M***; Sub-M > Unb-M*** \\
\hline
\end{tabular}

Anmerkungen: Ein Komma trennt gleichrangige Gruppen, ein Semikolon kennzeichnet den Beginn eines neuen Vergleichs. Größer- und Kleinerzeichen markieren die Richtung eines signifikanten Unterschieds.

${ }^{*}=\mathrm{p} \leq .05,{ }^{* *}=\mathrm{p} \leq .01,{ }^{* * *}=\mathrm{p} \leq .001$

hohen Risiko für Schwierigkeiten in familialen Interaktionen und Beziehungen einhergeht.

Bezüglich des Total-Stress-Wertes (Summe aus kind- und elternbezogenen Werten) bleibt festzuhalten, dass dieser für die Mütter mit einer Suchtstörung signifikant höher ausfällt als für die unbelasteten Mütter; gleichzeitig wird deutlich, dass die substituierten Mütter hier noch stärker belastet sind als die Alkoholikerinnen (Tab. 3).
Diskussion

Im Rahmen der vorliegenden Untersuchung wurde das Belastungserleben in der Kindererziehung von alkoholabhängigen Müttern und substituierten opiatabhängigen Müttern fokussiert und mit dem von nicht suchtbelasteten Müttern verglichen.

Zunächst bestätigen unsere Ergebnisse den akuten Unterstützungsbedarf von Müttern mit einer Suchtstörung, wie er zumindest von opiatabhängigen Müttern in vergleichbaren Untersuchungen auch schon selbst berichtet wurde [20]. Besonders deutlich wurde, dass suchtbelastete Mütter ihre eigenen erzieherischen Kompetenzen überaus negativ und defizitär einschätzen. Sie haben kaum Selbstvertrauen in ihre eigenen Fähigkeiten als Mutter, was sich nicht zuletzt auch in Unsicherheiten und Inkonsistenzen im konkreten Erziehungsverhalten niederschlagen dürfte. Gestützt wird diese Vermutung durch Untersuchungen, die darauf aufmerksam machen, dass Eltern mit niedrigen Kompetenzüberzeugungen eher ungünstiges Erziehungsverhalten zeigen, wie z.B. gewalthaltige Disziplinierungsversuche [21]. Darüber hinaus erreichen die suchtbelasteten Mütter im Bereich der sozialen Isolation besorgniserregend hohe Belastungswerte. Die fehlende soziale Integration und die ausgeprägten Einsamkeitsgefühle lassen darauf schließen, dass suchtbelastete Frauen kaum auf emotionale und instrumentelle Unterstützung bei der Erziehung ihrer Kinder zurückgreifen können. Abidin [17] betont, dass erhöhte Werte in diesem Bereich „Signalcharakter“ haben und dass die betroffenen Eltern so schnell wie möglich professioneller Hilfe zugeführt werden sollten. Die Dringlichkeit für den Interventionsbedarf ergibt sich hier vor allem daraus, dass eine extreme soziale Zurückgezogenheit und ein Fehlen unterstützender Sozialbeziehungen mit einer erhöhten Vernachlässigungsgefahr der betroffenen Kinder einhergehen [17].

Während die anhand des PSI ermittelten Stresswerte im Bereich der Elterndimensionen (Kompetenz, Isolation, Bindung, Gesundheit, Einschränkungen durch die Elternrolle, Depression und Partnerschaft) für die alkohol- und opiatabhängigen Mütter weitgehend parallel auf hohem Niveau mit der Tendenz zu höheren Werten für die substituierten drogenabhängigen Mütter verlaufen, zeigen sich auf den kindbezogenen Stressdimensionen zwischen diesen beiden Gruppen einige Unterschiede. Es wäre zu klären, ob die Differenzen hinsichtlich der „Anpassungsfähigkeit des Kindes“ und den „Anforderungen an die Eltern“ durch die ungünstigeren Lebensumstände opiatabhängiger Mütter (mehr allein Erziehende, weniger Erwerbstätige) oder die unterschiedliche Lebenssituation zum Zeitpunkt der Befragung (alkoholabhängige Mütter in Kliniken versus substituierte opiatabhängige Mütter in ihrem üblichen Lebensumfeld) zu erklären sind, oder ob deren Kinder in diesen Bereichen dauerhafte Unterschiede zeigen. Dadurch könnten Indikationen auch für kindbezogene Hilfen eruiert werden.

Insgesamt kann jedoch festgehalten werden, dass sich, neben dem unmittelbaren Hilfebedarf, ein ganz spezifisches Belastungsmuster für süchtige Mütter herauskristallisiert: Die Mütter betrachten in erster Linie eigene Defizite als Ursache von Erziehungsschwierigkeiten, d. h. Probleme in der Erziehung führen die Frauen überwiegend auf sich selbst - und weniger auf belastende oder auffällige Eigenschaften und Verhaltensweisen ihrer Kinder - zurück. 
Ein ganz zentrales Ergebnis ist, dass dieses Belastungsprofil sowohl kennzeichnend ist für alkoholabhängige Mütter als auch für opiatabhängige Mütter in Substitutionsbehandlung. Zwar ergibt sich im Hinblick auf einige kindbezogene Einschätzungen eine stärkere Belastung der opiatabhängigen Frauen, in der elternbezogenen PSI-Dimension beeindruckt jedoch die ausgeprägte Ähnlichkeit der Profilverläufe. Immerhin handelt es sich bei Alkoholund Opiatabhängigkeit um zwei unterschiedliche Suchterkrankungen, die in der Regel auch mit einem deutlich anderen Lebensstil und einer unterschiedlichen soziodemographischen Situation (Tab.1) verknüpft sind. Möglicherweise resultieren die starken Zweifel an den eigenen elterlichen Kompetenzen zu einem wesentlichen Anteil aus eigenen negativen bis traumatischen Kindheitserlebnissen - und die finden sich in den Biographien von Frauen mit jeglichen Suchterkrankungen [22, 23].

Es kann jedoch nicht abschließend eingeschätzt werden, wie realistisch die stark negativen Selbsteinschätzungen der alkohol- und opiatabhängigen Frauen sind. Einerseits könnten sie Ausdruck eines übertrieben negativen Selbstkonzepts sein, andererseits könnten sie selbst perzipierte, aber tatsächlich vorhandene Defizite widerspiegeln. Dass für die letztgenannte Annahme klare Belege vorliegen, verdeutlicht der umfangreiche Sammelbeitrag von Hogan [24], in dem zahlreiche derartige Defizite im Erziehungsverhalten - wie z.B. stärkeres Kontrollverhalten, häufigere aversive Äußerungen, stärkeres unangemessenes Schimpfen - aufgezeigt werden.

In praktisch-therapeutischer Hinsicht wirft die starke Übereinstimmung des erziehungsbezogenen Belastungserlebens zwischen alkohol- und opiatabhängigen Müttern die Frage auf, ob es folgerichtig ist, gemeinsame Elternkurse anzubieten. Insgesamt erscheint es doch günstiger, spezifische Unterstützungsangebote für die verschiedenen Suchterkrankungen durchzuführen, da es in der Regel einfacher ist, in homogenen Gruppen ein Klima der gegenseitigen Wertschätzung und Akzeptanz zu etablieren und aufrechtzuerhalten.

Abidin [17] empfiehlt außerdem, Interventionsangebote für Eltern mit stark beeinträchtigten Kompetenzgefühlen eher eltern- und weniger kindzentriert anzulegen. Um das niedrige Kompetenzerleben nicht noch weiter zu beschädigen, sollte darauf geachtet werden, nicht zu schnell Probleme oder Schwierigkeiten anzusprechen, die mit dem Erziehungsverhalten der betreffenden Eltern verbunden sind. Vielmehr sollte die gezielte Stärkung des elterlichen Selbstbewusstseins und die Förderung einer positiven Selbstwirksamkeitserwartung als Mutter im Zentrum entsprechender Interventionsangebote stehen.

Zusammengenommen verdeutlichen unsere Ergebnisse einmal mehr, dass es unerlässlich ist, spezifische Hilfsangebote für Mütter mit Suchterkrankungen zu entwickeln und in der Praxis der Suchtund Drogenhilfe sowie der Kinder- und Jugendhilfe zu etablieren.

\section{Literatur}

${ }^{1}$ Kreppner K. Persönlichkeitsentwicklung in sozialen Beziehungen. In: Asendorpf JB (Hrsg). Soziale, emotionale und Persönlichkeitsentwicklung. Enzyklopädie der Psychologie (Themenbereich C, Bd. 3). Göttingen: Hogrefe, 2005: 617-675

${ }^{2}$ Maccoby EE. Parenting and its effects on children: On reading and misreading behavior genetics. Annual Review of Psychology 2000; 51: $1-27$

${ }^{3}$ Steinberg L. We know some things: Parent-adolescent relationships in retrospect and prospect. Journal of Research on Adolescence 2001; 11: $1-19$

${ }^{4}$ Cummings EM, Goeke-Morey MC, Papp LM. Couple conflict, children, and families: It's not just you and me, babe. In: Booth A, Crouter AC, Clements M (Hrsg). Couples in conflict. Mahwah NJ: Lawrence Erlbaum, 2001: $117-147$

${ }^{5}$ Katz LF, Gottman JM. Patterns of marital conflict predict children's internalizing and externalizing behaviors. Developmental Psychology 1993; 29: $940-950$

${ }^{6}$ Wissenschaftlicher Beirat für Familienfragen. Familiale Erziehungskompetenzen. Beziehungsklima und Erziehungsleistungen in der Familie als Problem und Aufgabe. Weinheim: Juventa, 2005

${ }^{7}$ Bundesministerium für Familie, Senioren, Frauen und Jugend (Hrsg). Zwölfter Kinder- und Jugendbericht. Bericht über die Lebenssituation junger Menschen und die Leistungen der Kinder- und Jugendhilfe in Deutschland. Berlin: Internetredaktion des BMFSFJ, 2005

${ }^{8}$ Sanders MR, Ralph A. Familienintervention und Prävention bei Verhaltensstörungen im Kindes- und Jugendalter. In: Schlottke PF, Schneider S, Silbereisen RK, Lauth GW (Hrsg). Störungen im Kindesund Jugendalter - Grundlagen und Störungen im Entwicklungsverlauf. Enzyklopädie der Psychologie (Themenbereich D, Bd. 5). Göttingen: Hogrefe, 2004: $341-378$

${ }^{9}$ Tschöpe-Scheffler S. (Hrsg). Konzepte der Elternbildung - eine kritische Übersicht. Opladen: Budrich, 2005

${ }^{10}$ Englert E, Ziegler M. Kinder opiatabhängiger Mütter - Ein Überblick. Suchttherapie 2001; 2: 143 - 151

${ }^{11}$ Klein M. Alkohol und Familie: Forschung und Forschungslücken. In: Kruse G, Körkel J, Schmalz U (Hrsg). Alkoholabhängigkeit erkennen und behandeln. Bonn: Psychiatrie Verlag, 2000: 133-185

${ }^{12}$ Klein M. Kinder aus alkoholbelasteten Familien - Ein Überblick zu Forschungsergebnissen und Handlungsperspektiven. Suchttherapie $2001 ; 2: 118-124$

${ }^{13}$ Lachner G, Wittchen HU. Familiär übertragene Vulnerabilitätsmerkmale für Alkoholmissbrauch und -abhängigkeit. In: Watz H, Rockstroh B (Hrsg). Abhängigkeit und Missbrauch von Alkohol und Drogen. Göttingen: Hogrefe, 1997: $43-89$

${ }^{14}$ Bundeszentrale für gesundheitliche Aufklärung BzgA. Alkohol in der Schwangerschaft - Ein kritisches Resümee. Eine Expertise im Auftrag der BzgA von Hiltrud Merzenich und Peter Lang (Forschung und Praxis der Gesundheitsförderung Bd. 17). Köln: BzgA, 2002

${ }^{15}$ Scheib H, Steier M. Hilfen für drogenabhängige Eltern und deren Kinder. Endbericht der wissenschaftlichen Begleitung des Modellprojekts KiD Hilfe für Kinder von Drogenabhängigen. Frankfurt: Institut für Sozialarbeit und Sozialpädagogik ISS e.V, 1998

${ }^{16}$ Wusterack B. Das Erziehungsverhalten von alkoholkranken Müttern. Empirische Untersuchung in stationären Suchtfachkliniken. Unveröffentlichte Diplomarbeit. Katholische Fachhochschule NordrheinWestfalen, Abteilung Köln, 2003

${ }^{17}$ Abidin RF. Parenting Stress Index. Odessa: Psychological Assessment Resources, 1995

${ }^{18}$ Bortz J. Statistik für Sozialwissenschaftler. Berlin: Springer, 1999

${ }^{19}$ Hager W. Grundlagen einer Versuchsplanung zur Prüfung empirischer Hypothesen in der Psychologie. In: Lüer G (Hrsg). Allgemeine experimentelle Psychologie. Göttingen: UTB, 1987: 43-253

${ }^{20} \mathrm{Klemm}$-Vetterlein S. Modellprojekt Fachberater Methadon. Düsseldorf: Ministerium für Frauen, Jugend, Familie und Gesundheit des Landes Nordrhein-Westfalen, 2000

${ }^{21}$ Mash EJ, Johnston C, Kovitz K. A comparison of mother-child interaction of physically abused and non-abused children during a play and task situation. Journal of Clinical Child Psychology 1983; 12: $337-346$ 